\title{
Web Service
}

National Cancer Institute

\section{Source}

National Cancer Institute. Web Service. NCI Thesaurus. Code C45412.

A software-powered resource or functional component whose capabilities can be accessed at an internet URI; an automated resource accessed via the Internet. 